A Network Analysis of Facebook Use and Well-being in Relation to Key Psychological

\title{
Variables: Replication and Extension
}

Lien Faelens1*, , Eowyn Van De Putte1,2, Kristof Hoorelbeke1, Rudi De Raedt1, \& Ernst H.W. Koster1

1. Department of Experimental Clinical and Health Psychology, Ghent University, Henri Dunantlaan 2, B-9000, Ghent, Belgium

2. Light \& Lighting Laboratory, KU Leuven, Gebroeders De Smetstraat 1, 9000 Gent, Belgium

* Corresponding author at: Department of Experimental Clinical and Health Psychology, Ghent University, Henri Dunantlaan 2, B-9000, Ghent, Belgium. Phone: +32 (0)9 26494 14; Fax: +32 (0)9 2646489

E-mail address: lien.faelens@ugent.be 


\begin{abstract}
Studies exploring the relationship between Facebook use and well-being have yielded inconsistent findings. Investigating the underlying mechanisms seems to be of crucial importance to gain insight into the positive and negative consequences of Facebook use. A recent study illustrated the importance of taking into account social comparison and selfesteem, which serve as central constructs in the relationship between Facebook use and risk for affective disorders. Extending these findings will be key to increase our knowledge on possible risk and/or protective mechanisms. Therefore, we conducted a cross-sectional study $(n=469)$ to investigate the position of attention control and social capital in this previous reported network. Our results provide a conceptual replication of Faelens et al. (2019). In addition, the findings suggest that attentional control is not strongly connected with the problematic effects of Facebook use. Importantly, (bridging) social capital uniquely connected the variables related to Facebook use with our indicators of vulnerability for affective disorders via social comparison and contingent self-esteem. Possible explanations are discussed.
\end{abstract}

Keywords: Social media, Facebook Social Capital, Social Comparison, Self-esteem, Attentional Control, Depression, Anxiety, Stress 


\section{Introduction}

In modern society, social network sites (SNS) play a central role in our daily lives. This has generated a lively debate regarding the positive and negative effects of SNS use on general well-being. Studies that focused on the relationship between SNS use and well-being found that SNS use is related to elevated depressive and/or anxiety symptoms (Woods \& Scott, 2016). However, prior studies have not been consistent in observing this relationship (Steers, 2016). As such, in order to shed further light on the relationship between SNS use and well-being, the literature would benefit from a more thorough focus on potential factors that determine consequences of social media use.

Importantly, a recent study in which network analysis was used to model the unique pathways between Facebook use and risk for affective disorders, suggested a crucial role for social comparison and self-esteem (Faelens, Hoorelbeke, Fried, De Raedt \& Koster, 2019). In particular, it has been suggested that the strategically presented content, by which posts are selected to create a more favorable impression to others, could trigger social comparison and induce negative self-evaluations (Vogel, Rose, Roberts, \& Eckles, 2014; Vogel \& Rose, 2016). Repeated exposure to such content could over time lower global self-esteem and eventually foster more detrimental psychopathological processes (Sowislo \& Orth, 2013; Wouters et al., 2013). Although these initial findings offer interesting insights into the relation between Facebook use and well-being, extending this network model with cognitive and social parameters will be key to further improve our understanding of this complex relationship. Therefore, the goal of the current study was to investigate the role of attentional control and social capital in relation to Facebook use, social comparison, (contingent) self-esteem, and risk for affective disorders (rumination, stress-, depressive- and anxiety symptoms). In particular, we modeled the impact of these variables on the network structure that was previously obtained 
by Faelens et al. (2019; Figure 1). In particular, the choice for these constructs was based on the following arguments.

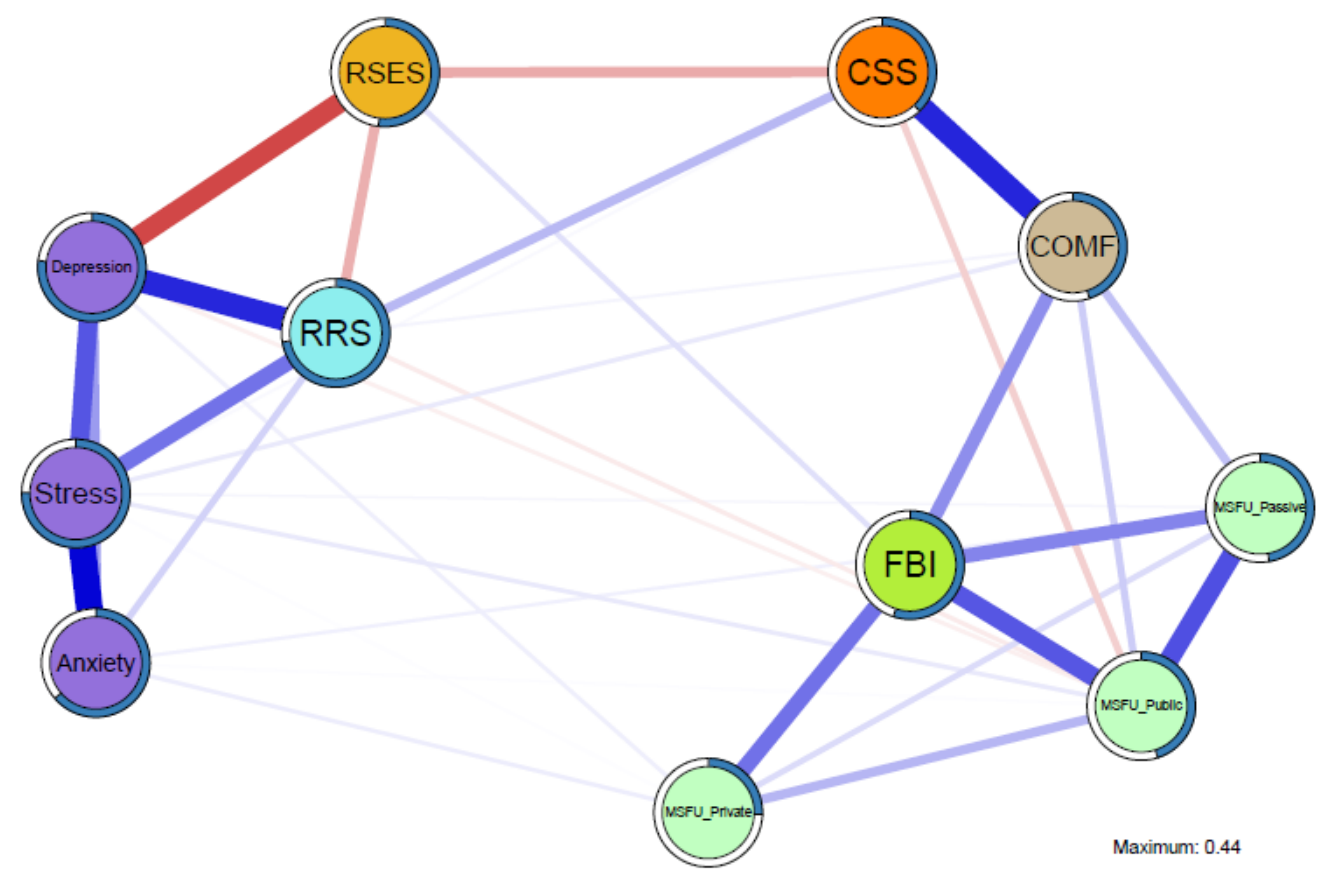

Figure 1. Regularized Partial Correlation Network Faelens et al. (2019)

FBI $=$ Facebook intensity; MSFU.PR = active private Facebook use; MSFU.PU = active public Facebook use; MSFU.PA = passive Facebook use; COMF = social comparison; CSS = contingent self-esteem; RSES = global self-esteem; RSS = ruminative tendency; STRESS = stress; ANX = anxiety; DEPR = depression

\subsection{Attentional Control}

Previous studies suggest that highly frequent use of social media, and the extent to which this relies on multitasking, could be detrimental for our ability to control our attention. Specifically, it has been suggested that people who often engage in social media multitasking may be more susceptible to interference of irrelevant information due to reduced attentional control (Bermúdez, 2017).

For example, Ophir, Nass \& Wagner (2009) showed that heavy social media multitaskers performed worse on a switching task than low social media multitaskers. These results suggest that heavy social media multitaskers are more likely to experience difficulties to block out distractions and focus their attention on a single task. This is in accordance with the 
counterintuitive notion that people who are not capable of multitasking efficiently are in fact the people who are most likely to engage in multiple tasks simultaneously (Sanbonmatsu, Strayer, Medeiros-Ward, \& Watson, 2013). Related to this, it is also important to consider the impact of social media multitasking on academic performance given that attention control plays a crucial role in learning. Indeed, previous studies suggest that people who spent more time using social media while attending classes, studying and doing homework, had lower comprehension of the lecture material and lower grades (Junco \& Cotton, 2011; Junco \& Cotton, 2012; Kirschner \& Karpinski, 2010; Gupta \& Irwin, 2016; Rosen, Mark Carrier, \& Cheever, 2013; Wood, 2012).

The disadvantageous impact of chronic social media use on academic performance and attention control is likely mitigated by multitasking (Chen \& Yan, 2016; Junco, 2015). However, note that the negative influence of social media multitasking on attention control also seems to depend on task difficulty. The negative influence of social media multitasking on attention control mainly seems to hold for complex tasks (Min, 2017).

Furthermore, ample studies suggest that attentional control deficits may put one at risk for developing depressive symptomatology (for reviews, see De Raedt \& Koster, 2010; Joormann, Talbot, \& Gotlib, 2007; Joormann \& Gotlib, 2010). In particular, depressed individuals seem to have difficulties focusing their attention towards positive information and relocating their attention away from negative towards positive information (Armstrong \& Olatunji, 2012). Moreover, these deficits in attentional control have been linked to emotion regulation difficulties, which are known to impact the onset and maintenance of depressive symptoms (Joormann \& Stanton, 2016). Together, these findings point towards the importance of extending previous network models with attentional control in an attempt to further unravel pathways between social media use and self-reported psychopathology (e.g., depressive, 
anxiety, and stress symptoms). A second variable that would be worthwhile investigating in this context, is social capital.

\subsection{Social Capital}

The desire to form and maintain interpersonal relationships is a fundamental human motivation (Baumeister \& Leary, 1995). Not surprisingly, research has shown that having positive relationships increases our well-being and visa versa (Lyubomirsky, King, \& Diener, 2005). For example, Sherman, Lansford and Volling (2006) illustrated that participants who had harmonious relationships with their family and friends (warm relationships with little conflict) reported lower loneliness and higher self-esteem scores. Furthermore, Sousa-Poza and SousaPoza (2000) suggested that having a good relationship with your colleagues and managers is an important determinant of job satisfaction. Indeed, social networks are important for people, since users derive numerous benefits from our social relations or connections, which are often described as 'social capital'. Putnam (2000) makes a distinction between 'bonding social capital' and 'bridging social capital'. Bonding social capital is typically provided by emotionally close relationships, such as family members and good friends. These individuals tend to share similar backgrounds and usually provide emotional/social support, trust and companionship to each other. Alternatively, having a large amount of 'weak ties' or acquaintances, which are casual connections between individuals who travel in different circles, provides bridging social capital. These connections give individuals access to novel information and a broader worldview, since they are more likely to add non-redundant information and new perspectives, not possessed by the individual's family or friends. Consequently, such relations do not necessarily provide emotional support.

SNS such as Facebook have the potential to foster communication with our close and weak connections. Indeed, SNS are particularly well-suited to maintain existing relationships, and to keep up to date with Facebook friends and therefore provide social capital benefits (Ellison et 
al., 2007). For example, Phua \& Jin (2011) highlighted that intensity of Facebook use contributed to bonding social capital. More specifically, elevated social support might reduce the negative impact of social media use on well-being and could in some circumstances even lead to improved well-being (Burke \& Kraut, 2013; Verduyn et al., 2017). Therefore, we expected that bonding social capital could possibly serve as an underlying protective mechanism in the relationship between Facebook use and well-being

In addition, SNS provide users the opportunity to activate latent ties into weak or bridging ties. This gives users the chance to maintain connections that would otherwise disappear, allowing (intensive) users to maintain larger and more heterogeneous networks (Brandtzaeg, 2012; Ellison et al., 2007). More specifically, these networks provide access to novel information and diverse viewpoints (e.g., via profile browsing, tagging, ... ; Burke et al., 2010). Furthermore, individuals who use Facebook at least partly for information seeking, reported higher scores on bridging social capital. Information seekers were also more likely to agree with the fact that they use this channel to 'check out' someone they met in a social environment, to learn more about them. In this way, Facebook can facilitate (offline) social interactions and therefore lead to positive consequences (Burke, Kraut, \& Marlow, 2011). However, at the same time these individuals will be exposed to strategically presented information of individuals they don't know (well), which may induce upward comparison and negative self-evaluations (Vogel, et. al. 2014; Vogel \& Rose, 2016). Therefore, we wanted to explore whether bridging social capital would function as risk- rather than protective factor in the current network study.

\subsection{Current study}

The current study aims to investigate the impact of attentional control and social capital (bonding, bridging) on the network structure obtained by Faelens et al. (2019). This network showed complex associations between social media use, social comparison, self-esteem and 
indicators of risk for affective disorders. In particular, to improve our understanding of the complex relationship between Facebook use and risk for internalizing psychopathology, we added two additional constructs: social capital and attentional control. As such, our goal was threefold:

(1) to replicate the network structure obtained by Faelens et al (2019)

(2) to investigate the role of attentional control within this network

(3) to investigate the possible protective role of bonding social capital (3A) and bridging social capital within this network (3B)

\section{Methods}

\subsection{Participants}

We recruited 509 Facebook users aged 18-35 via Prolific Academic. Forty participants were excluded as they failed to answer reading check items correctly, resulting in a final sample of 469 participants. Participants provided informed consent prior to completing the survey. This study was approved by the local ethical committee.

\subsection{Measures}

Facebook Use. The Multidimensional Scale of Facebook Use (MSFU; Frison \& Eggermont, 2015) consists of 10 items, each rated on a 7-point Likert scale ranging from 1 ("never") to 7 ("several times per day"). This instrument measures three types of Facebook activities: passive Facebook use (MSFU.PA, e.g. "How often do you look at photos of a Facebook friend?"), active private Facebook use (MSFU. PR; e.g., "How often do you send a private message?"), and active public Facebook use (MSFU.PU; e.g., “How often do you post a status update?”). However in line with previous research, we decided to exclude one item of the passive Facebook use subscale (“How often do you read your news feed?") because this item loaded 
highly on another subscale (i.e., active private Facebook use; Faelens et al., 2019; Frison \& Eggermont, 2015). The internal consistency of the three subscales in the current study was as follows: passive Facebook use $(\alpha=.90)$, active private Facebook use $(\alpha=.90)$, and active public Facebook use $(\alpha=.91)$.

Facebook intensity. The Facebook Intensity Scale (FBI; Ellison, Steinfield, \& Lampe, 2007) assesses people's emotional connection to Facebook and its integration in people's daily lives. In this study, we only used the six attitudinal items, rated on a 5-point Likert scale (e.g., "Facebook has become part of my daily routine"). The scale showed good internal reliability (Cronbach's $\alpha=.88)$.

Facebook-specific bridging and bonding social capital. The Facebook-specific social capital measure (Su \& Chan, 2017) was adapted from Ellison, Vitak, Gray, and Lampe (2014). The Facebook-specific bridging social capital subscale (BRSC) consists of seven items and measures the degree to which people perceive bridging benefits (e.g. information that triggers new interests) from interaction with their Facebook friends (e.g. "Interacting with Facebook friends makes me interested in things that happen outside of my city", $\alpha=.89$ ). In contrast, the Facebook-specific bonding social capital subscale (BOSC) assesses the extent to what individuals report to experience bonding social capital benefits as emotional support and mutual trust via their Facebook friends (e.g. "There are several Facebook friends I trust to help solve my problems", $\alpha=.88$ ). Both subscales are rated on a 5-point Likert scale ranging from 1 ("strongly disagree) to 5 ("strongly agree).

Social comparison on Facebook. Social comparison on Facebook was measured with the Comparison Orientation Measure-Facebook (Steers, Wickham, \& Acitelli, 2014). The COM-F consists of 11 items and is an adaptation of the well-established Iowa-Netherlands Comparison Orientation Measure (Gibbons \& Buunk, 1999). All items were rated on a 5-point Likert scale 
(e.g., "When I am on Facebook, I try to find out what others think who face similar problems as I face", $\alpha=.88)$.

Contingent Self-esteem. The Contingent Self-Esteem Scale (CSS; Paradise \& Kernis, 1999) is a measure of global self-esteem contingency. The 15 -item instrument consists of 15 items each rated on a 5-point Likert scale ranging from 1 ("not at all like me") to 5 ("very much like me"; e.g., "An important measure of my worth is how physically attractive I am.") and has a good reliability $(\alpha=.83)$.

Global Self-esteem. The Rosenberg Self-Esteem Scale (RSES; Rosenberg, 1965) is a widely used measure of global feelings of self-worth and shows good reliability $(\alpha=.90)$. The 10 -item instrument instructs participants to rate how much they agree with each statement (e.g., "I take a positive attitude toward myself"), using a 4-point Likert scale ranging from 1 ("strongly disagree") to 4 (“strongly agree").

Attentional Control. We used the attentional control subscale of the Adult temperament questionnaire-Effortful control to assess attentional control (ATC; Evans \& Rothbart, 2007). This questionnaire consists of 5 items measured on a 7-point Likert scale ranging from 1 ("extremely untrue of you") to 7 ("extremely true of you"; e.g., "It's often hard for me to alternate between two different tasks", $\alpha=.68$ ).

Rumination. The Ruminative Responses Scale (RRS; Treynor, Gonzalez, \& Nolen-Hoeksema, 2003) is a 22-item instrument that measures trait rumination and indicates how often participants generally engage in repetitive negative thinking $(\alpha=.94)$. Responses are scored on a four-point Likert scale ranging from 1 (“almost never") to 4 (“almost always").

Negative Emotional States of Depression, Anxiety, and Stress. The Depression, Anxiety and Stress Scales (Lovibond \& Lovibond, 1995) is a self-report questionnaire designed to measure psychological distress on three 7-item dimensions. Participants are instructed to report the extent to which they experience depressive- (DEPR; e.g., "I found it difficult to work up the 
initiative to do things", $\alpha=.92$ ), anxiety- (ANX; e.g., "I was worried about situations in which I might panic and make a fool of myself", $\alpha=.86$ ), and stress symptoms (STRESS; e.g., "I felt that I was using a lot of nervous energy; $\alpha=.87$ ) with response options ranging from 0 ("Did not apply to me at all") to 3 ("Applied to me very much, or most of the time").

\subsection{Data analyses}

Data analysis was conducted in $\mathrm{R}$ version 3.5.0 (see supplemental material for Rpackages used and version information). After detecting skew in the data, we conducted a nonparanormal transformation using the huge package (Zhao et al., 2015) to improve normality. In line with Faelens et al. (2019), we estimated a Gaussian Graphical Model (GGM; Epskamp \& Fried, 2018) using qgraph (Epskamp, Cramer, Waldorp, Schmittmann, \& Borsboom, 2012). We relied on partial correlations to model the unique shared variance between different nodes in the model, using regularization to remove spurious edges and maximize model specificity. In particular, we implemented regularization based on the Graphical Least Absolute Shrinkage and Selection Operator (gLASSO; Friedman, Hastie, \& Tibshirani, 2014) with Extended Bayesian Information Criterion model selection (EBIC; $\gamma=0.5$ ). Thresholding was used to maximize model specificity. We then estimated node predictability - the percentage of variance of each node that is explained by its neighbouring nodes in the network - with $m g m$ (Haslbeck \& Waldorp, 2016), and proceeded with bootstrapping procedures to assess the reliability of the obtained network model. In particular, bootnet (Epskamp \& Fried, 2017; Epskamp, Borsboom, $\&$ Fried, 2018) was used to compute the accuracy of the edge weights, where we provided $95 \%$ confidence intervals for all edges in the model. In addition, we plotted significant differences between edges, and estimated the stability of strength centrality. Node strength provides an estimate of the sum of absolute edge weights connected to each node. As such, node strength reflects strength of connectivity for a given node (Costantini et al., 2015). We standardized this 
centrality measure to a normal $\mathrm{Z}$ distribution to facilitate interpretation. Stability of the order of node strength within subsets of the data was established using a case-dropping subset bootstrap. In order to be considered stable, the resulting correlation stability coefficient should not be below 0.25 and preferably exceed 0.50 (Epskamp et al., 2018). We relied on a modification of the Fruchterman-Reingold's algorithm (Fruchterman \& Reingold, 1991) to visualize the network model. As such, nodes are positioned in the model based on their connectivity, with strongly connected nodes holding a more central position in the model than less connected nodes. The unique associations between two given nodes are represented by edges, where (a) the thickness of the edge reflects the strength, and (b) the type of line used and colour of the edge reflect the valence of this association (blue / full line $=$ positive, red $/$ dashed line $=$ negative $)$. Finally, node predictability was plotted as a pie chart in the outer ring of each node (Haslbeck \& Fried, 2017).

\section{Results}

Descriptive statistics of the variables of interest are reported in Table 1. Figure 1 depicts the GGM, suggesting two conceptual clusters related to use of Facebook (Facebook intensity (FBI), active public, passive and active private Facebook use (MSFU-PU/MSFU-PA/MSFUPR), bonding social capital (BOSC) and bridging social capital (BRSC)) and emotional vulnerability (rumination (RRS), depression (DEPR), stress (STRESS), anxiety (ANX), attentional control (ATC)). These clusters are indirectly linked via indicators of social comparison (COMF) and (contingent) self-esteem (CSS). Within the cluster related to Facebook use, Bridging social capital (BRSC) emerged as a bridging construct, being connected to most indicators of Facebook use and social comparison (COMF). Within the cluster of emotional vulnerability, attentional control (ATC) showed unique negative associations with stress and rumination. The latter emerged as the most strongly connected node in the model, closely followed by stress (STRESS), depression (DEPR), Facebook intensity (FBI) and 
bridging social capital (BRSC). Based on node strength, attentional control (ATC) was the least central node in the model (Figure 2). This was also reflected in terms of node predictability. For the corresponding weight matrix and indicators of node predictability, see supplemental Tables 1 and 2.

The obtained model showed acceptable accuracy and stability, as indicated by the bootstrapped 95\% confidence intervals around the edge weights (Supplemental Figure 1) and the obtained correlation stability coefficient for Strength centrality (.60). That is, with a 95\% probability, a maximum of $60 \%$ of the original sample could be dropped while remaining a correlation $\geq .70$ for Strength centrality between the original sample and the obtained samples following the case-dropping subset bootstrapping procedure (Supplemental Figure 2). Significant differences between edge weights are plotted in Supplemental Figure 3. 
Table S1. Sample Characteristics

\begin{tabular}{|c|c|}
\hline & $N=459$ \\
\hline Female: Male & $232: 227$ \\
\hline \multicolumn{2}{|l|}{ Continent } \\
\hline North America & 52 \\
\hline South America & 2 \\
\hline Europe & 392 \\
\hline Africa & 0 \\
\hline Asia & 8 \\
\hline Oceania & 4 \\
\hline Antarctica & 1 \\
\hline Age & $26.20(4.525)$ \\
\hline Facebook intensity (FBI) & $18.46(5.73)$ \\
\hline Social comparison (COMF) & $33.63(8.70)$ \\
\hline Global self-esteem (RSES) & $17.46(5.54)$ \\
\hline Ruminative tendency (RRS) & $49.12(14.02)$ \\
\hline Contingent self-esteem (CSS) & $51.40(8.13)$ \\
\hline Passive Facebook use (MSFU.PA) & $13.69(5.06)$ \\
\hline Active private Facebook use (MSFU.PR) & $9.07(3.80)$ \\
\hline Active public Facebook use (MSFU.PU) & $7.09(3.54)$ \\
\hline Stress (STRESS) & $7.37(4.84)$ \\
\hline Anxiety (ANX) & $5.06(4.65)$ \\
\hline Depression (DEPR) & $6.39(5.58)$ \\
\hline Attentional control (ATC) & $3.99(1.12)$ \\
\hline Bridging social capital (BRSC) & $21.17(5.85)$ \\
\hline Bonding social capital (BOCS) & $17.54(5.72)$ \\
\hline
\end{tabular}

Note: Standard deviations are given in parentheses. 


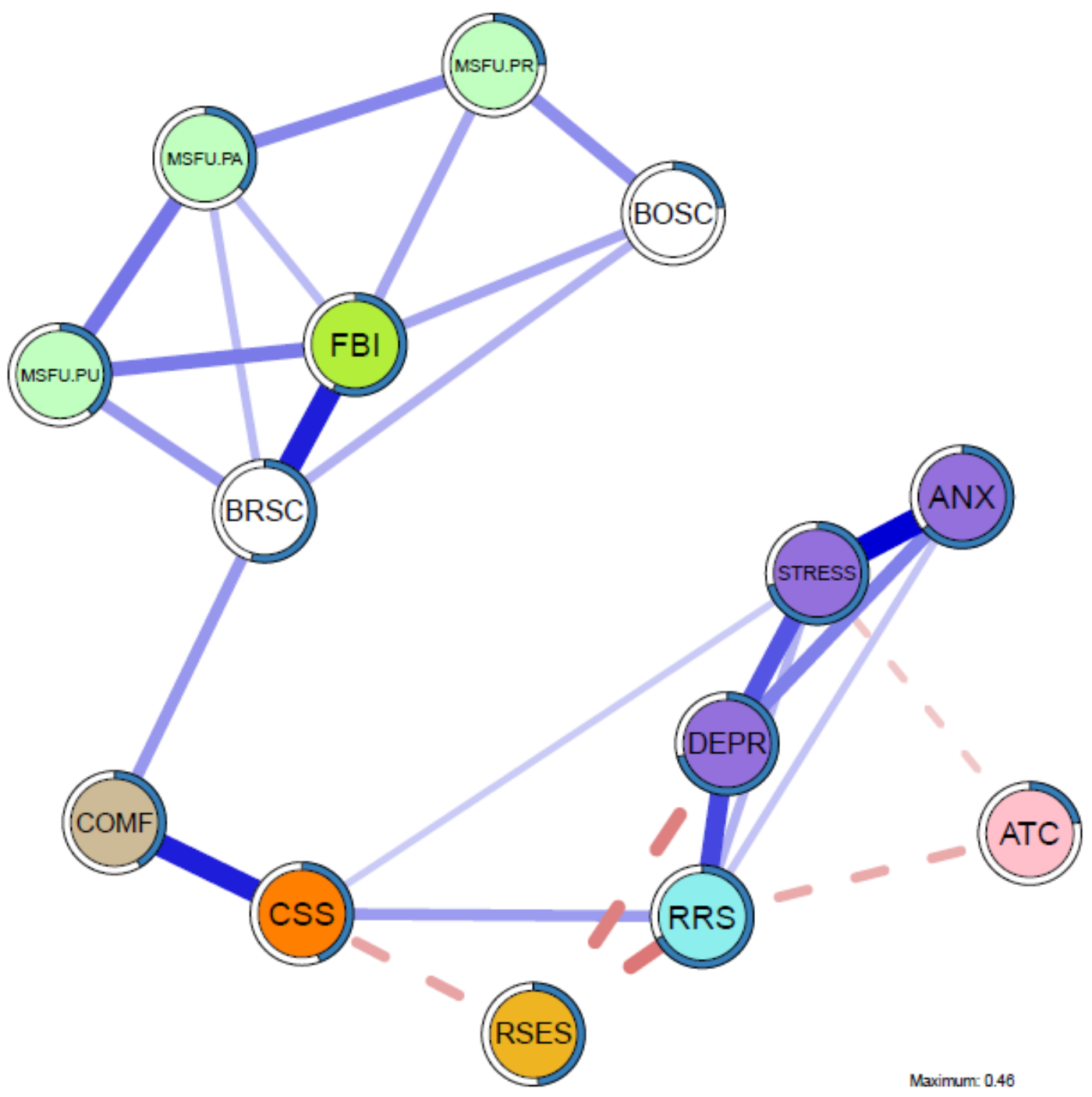

Figure 2. Regularized Partial Correlation Network

FBI = Facebook intensity; MSFU.PR = active private Facebook use; MSFU.PU = active public Facebook use; MSFU.PA = passive Facebook use; BOCS = bonding social capital; BRSC = bridging social capital; $\mathrm{COMF}=$ social comparison; $\mathrm{CSS}=$ contingent self-esteem; RSES = global self-esteem; RSS = ruminative tendency; ATC = attentional control; STRESS = stress; ANX = anxiety; DEPR = depression 


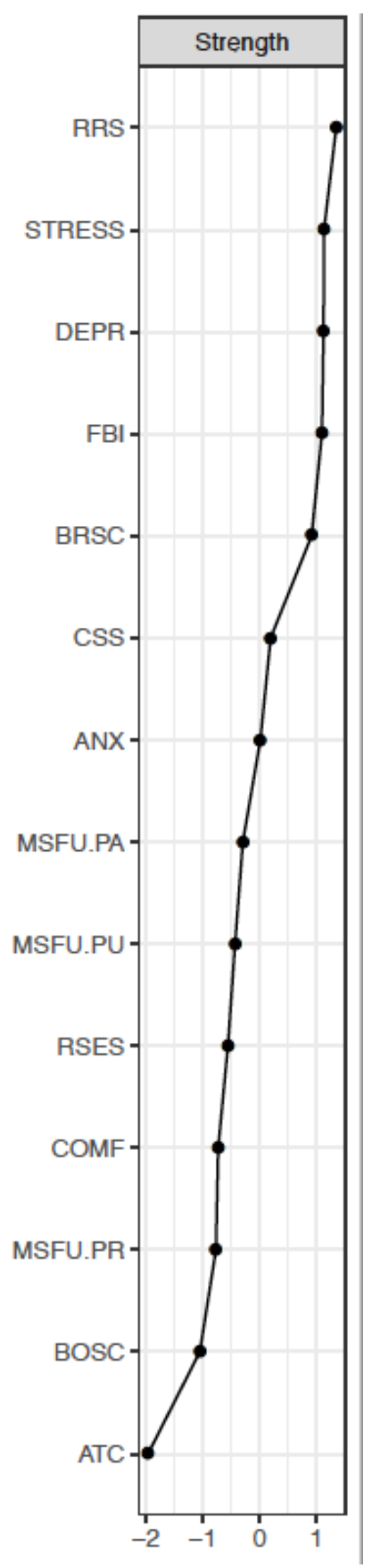

Figure 3. Strength Centrality

\section{Discussion}

The goal of the current study was to model the complex interrelations between Facebook use, social capital, social comparison, self-esteem, attentional control, and indicators of risk for affective disorders (level of stress-, anxiety-, and depressive symptomatology). In this context, we used network analysis to provide a comprehensive overview of associations between these 
constructs. The main findings are that: (1) the results provide a conceptual replication of Faelens et al. (2019); (2) attentional control is not strongly associated with the problematic effects of Facebook use; and (3A) bonding social capital does not occupy a central position in the network nor functions as a protective factor. (3B) Bridging social capital, however, plays a central role in linking Facebook use and well-being via social comparison and (contingent) self-esteem. We discuss each of these findings in turn.

First, our results provide support for the importance of social comparison and selfesteem, connecting Facebook use with indicators of psychopathology. In line with our expectations, the obtained network structure is highly similar to the model obtained by Faelens et al. (2019). That is, the Facebook cluster was linked to social comparison behavior and social comparison linked Facebook use with self-esteem. In turn, self-esteem connects the indicators of psychopathology with social comparison and indicators of Facebook use.

Second, we found no direct link between Facebook use and attentional control as measured with the effortful control scale. Although attentional control did not take a central mediating role in connecting Facebook use with depression, it could still have an influence as indirect links emerged via rumination, self-esteem, and social comparison. That is, individuals showing poor attentional control may be more prone to ruminate, which increases the likelihood of experiencing depressive-, stress-, or anxiety symptoms (Koster, De Lissnyder, Derakshan \& De Raedt, 2011).

Related to this, ruminators may report lower self-esteem and be more likely to compare themselves on Facebook. Vice versa, Facebook use may trigger social comparison, which can have a detrimental impact on self-esteem and may induce rumination, resulting in poor attentional control. This pathway provides evidence for the impulsivity pathway proposed by Billieux (2012). This pathway states that people who score high on the impulsivity personality 
trait are more likely to start ruminating when they are confronted with negative feelings due to reduced self-control. As a consequence social media use might serve as a short-term avoidance strategy to deal with unpleasant emotions to get rid of the ruminative thoughts.

Furthermore, there are also several tentative explanations for the absence of direct links between attentional control and problematic effects of Facebook use. First, attentional control can fluctuate over time, but the ATQ only measures attention as a stable construct. Hence, important fluctuations in attention are not captured within the current approach. Second, individuals might not be able to give an accurate estimate of their attentional capacities in the face of distracting information.

In addition to extending the initial model with attentional control (cf. Faelens et al., 2019), we also examined the role of bonding and bridging social capital. In line with previous research, both constructs are directly connected with Facebook intensity (Ellison et al., 2007), suggesting that people who feel strongly connected to this platform experience higher social capital outcomes. As expected by their different nature, both constructs show unique associations in the network. First, bonding social capital is uniquely associated with active private Facebook use, which is in line with previous research showing that one-on-one communication (e.g., via instant messaging) with friends is associated with social capital benefits as perceived social support (Frison \& Eggermont, 2016). Next, we hypothesized that social capital could act as a protective factor within our network. In contrast to the pattern of results reported in the review of Verduyn et al. (2017), we found no direct link between Facebook use and indicators of well-being. One possible explanation for this finding is that we mainly included negative outcome measures such as stress, anxiety and depressive symptoms. Previous research has shown that feelings of social support and relatedness are mainly linked to positive outcomes instead of negative outcomes (Reis, Sheldon, Gable, Roscoe, \& Ryan, 2018). For example, Watson and Clark (1994) showed that positive affect is raised when people 
are socializing, whereas negative affect is primarily a function of stressful or aversive events. Another possible explanation is that we focused on Facebook-specific social capital and did not include a measure of offline social capital. Previous research has shown that computer-mediated support especially helps people who are unable to connect with others in an offline environment. As an illustration, for socially anxious individuals, online social support via Facebook provided an additional source of contact above offline social support, increasing subjective well-being. However, this beneficial effect of additional online social contacts did not contribute to well-being in the low anxiety group (Indian \& Grieve, 2014). This may explain the absence of a direct connection between Facebook-specific bonding social capital and indicators of well-being in our healthy convenience sample.

In contrast, bridging social capital emerged as a key construct. That is, bridging social capital seems to connect (a) intensity of Facebook use, active public Facebook use, and passive Facebook use, with (b) social comparison, which provides support for the role of bridging social capital as potential risk factor in a social media context. The network shows that users, who engage in active interaction with their Facebook friends (e.g., via wall comments, tagging...) and are frequently looking at the content of their friends via profile browsing, tend to have a higher bridging social capital. However, looking at the strategically presented content of others may facilitate the process of social comparison with their acquaintances. Although these users have access to a lot of new information due to their heterogeneous network, bridging social capital thus can have some unexpected downsides. As users with a higher bridging social capital may perceive more desirable content of others., this can be accompanied by negative selfevaluations when users rely on this extensive network to compare themselves to others, which may then induce psychopathology symptoms (Vogel \& Rose, 2016). Vice versa, being in a negative state with low self-esteem may trigger more social comparison and bridging social capital. 
The current study set out to model the unique associations between central indicators of Facebook use and vulnerability for affective disorders. An important strength of this study is that it examines the role of both social capital and social comparison in the relationship between Facebook use and indicators of well-being. Previous studies suggested that these constructs could be important mechanisms underlying the effects of Facebook use and well-being. For example, they reported that browsing on Facebook could cause distress, by inducing social comparison and envy. In contrast, active communication with Facebook friends could increase people's social capital, which results in a higher well-being. However, in the current study we only found support for the role of social comparison as possible underlying mechanisms in the negative relationship between Facebook use and well-being. With regard to the positive relationship, we did not find a direct link between social capital and indicators of psychopathology. Second, we were one of the first to model the unique associations of attentional control in the context of Facebook use and psychopathology. This is of particular interest given the well-established role of attentional control in emotion regulation processes (De Raedt \& Koster, 2010; Joormann, Talbot, \& Gotlib, 2007; Joormann \& Gotlib, 2010; Joormann \& Stanton, 2016) and previously reported inconsistencies pertaining the relation between social media use and attentional control (Chen \& Yan, 2016; Junco, 2015; Min, 2017; Ophir, Nass \& Wagner, 2009).M0

The findings of the present study are limited in some respects. Due to the use of a convenience sample that we recruited via Prolific Academic, the findings of this research should not be overgeneralized. Future research should extend this sample in order to map the relationships between Facebook use and indicators of psychopathology in a broader group of Facebook users in terms of age groups, ethnicity, etc. Second, the present study was limited by self-report measures. Future studies should include more objective measurements of Facebook use (e.g., software) and indicators of stress, anxiety and depression (e.g. cortisol measurements). Third, 
it should be noted that the obtained network model stems from cross-sectional data and is undirected. As such, no inferences can be made regarding causality and the direction of the observed relationships. Based on the existing literature - these findings likely represent bidirectional pathways. The key strength of this exploratory data-driven approach is that it allows to identify and model potential mechanisms across a broad range of constructs. However, the direction and causality should be further tested using prospective or experimental designs (e.g., Aalbers, McNally, Heeren, de Wit, \& Fried, 2018)

\section{Conclusion}

This study set-out to replicate and extend the network model obtained by Faelens et al. (2019) with two factors: (1) attentional control and (2) social capital. Our results provide a conceptual replication and extension of the network model(s) obtained by Faelens et al. (2019). Interestingly, attentional control showed no direct associations with Facebook use, whereas bridging social capital emerged as a key variable in the network uniquely connecting indicators of (intensity of) Facebook use with indicators of risk for affective disorders via social comparison and self-esteem. These findings advance our understanding regarding the complex relation between Facebook use and psychological well-being.

\section{Declaration of Interest}

There are no known conflicts of interest associated with this publication

\section{Funding}

This research was funded by a Special Research Fund (BOF) of Ghent University awarded to Lien Faelens (BOF16/DOC/323). Kristof Hoorelbeke is a Postdoctoral Fellow of the Research Foundation-Flanders (FWO; FWO.3EO.2018.0031.01). 


\section{Author contribution}

Lien Faelens and Eowyn Van De Putte developed the study concept and study design under the supervision of Ernst H.W. Koster and Rudi De Raedt. Kristof Hoorelbeke performed the data analysis and interpretation. Lien Faelens and Eowyn Van De Putte drafted the introduction section. Lien Faelens drafted the method and discussion section. Kristof Hoorelbeke drafted the results section. Rudi De Raedt and Ernst H.W. Koster provided critical revision. All authors approved the final version of the manuscript for submission. 


\section{References}

Aalbers, G., McNally, R. J., Heeren, A., de Wit, S., \& Fried, E. I. (2019). Social media and depression symptoms: A network perspective. Journal of Experimental Psychology: General, 148(8), 1454-1462. doi:10.1037/xge0000528

Armstrong, T., \& Olatunji, B. O. (2012). Eye tracking of attention in the affective disorders: A meta-analytic review and synthesis. Clinical psychology review, 32(8), 704-723.

Baumeister, R. F., \& Leary, M. R. (1995). The need to belong: desire for interpersonal attachments as a fundamental human motivation. Psychological bulletin, 117(3), 497.

Bermúdez, J. P. (2017). Social media and self-control: The vices and virtues of attention. Social Media and Your Brain, 57-74.

Billieux, J. (2012). Problematic use of the mobile phone: a literature review and a pathways model. Current Psychiatry Reviews, 8(4), 299-307.

Burke, M., Marlow, C., \& Lento, T. (2010). Social network activity and social well-being. In Proceedings of the SIGCHI conference on human factors in computing systems (pp. 1909-1912). ACM.

Burke, M., \& Kraut, R. (2013). Using Facebook after losing a job: Differential benefits of strong and weak ties. Conference on Computer-Supported Cooperative Work \& Social Computing, 1419-1430.

Burke, M., Kraut, R., \& Marlow, C. (2011). Social capital on Facebook: Differentiating uses and users. In Proceedings of the SIGCHI conference on human factors in computing systems (pp. 571-580). ACM.

Brandtzæg, P. B. (2012). Social networking sites: Their users and social implications-A longitudinal study. Journal of Computer-Mediated Communication, 17(4), 467-488.

Chen, Q., \& Yan, Z. (2016). Does multitasking with mobile phones affect learning? A review. Computers in Human Behavior, 54, 34-42. 
Costantini, G., Epskamp, S., Borsboom, D., Perugini, M., Mottus, R., Waldorp, L. J., Cramer, A. O. J. (2015). State of the aRt personality research: A tutorial on network analysis of personality data in R. Journal of Research in Personality, 54, 13-29. doi: 10.1016/j.jrp.2014.07.003

De Raedt, R., \& Koster, E. H. (2010). Understanding vulnerability for depression from a cognitive neuroscience perspective: A reappraisal of attentional factors and a new conceptual framework. Cognitive, Affective, \& Behavioral Neuroscience, 10(1), 50-70.

Ellison, N. B., Steinfield, C., \& Lampe, C. (2007). The Benefits of Facebook "Friends:" Social Capital and College Students' Use of Online Social Network Sites. Journal of Computer-Mediated Communication, 12(4), 1143-1168. doi:10.1111/j.10836101.2007.00367.x

Ellison, N. B., Vitak, J., Gray, R., \& Lampe, C. (2014). Cultivating Social Resources on Social Network Sites: Facebook Relationship Maintenance Behaviors and Their Role in Social Capital Processes. Journal of Computer-Mediated Communication, 19(4), 855-870.

Epskamp, S., Cramer, A., Waldorp, L., Schmittmann, V. D., \& Borsboom, D. (2012). qgraph: Network visualizations of relationships in psychometric data. Journal of Statistical Software, 48, 1-18. doi: 10.18637/jss.v048.i04

Epskamp, S., \& Fried, E. I. (2017). bootnet: Bootstrap methods for various network estimation routines. $\mathrm{R}$ package version 1.2 .

Epskamp, S., \& Fried, E. I. (2018). A Tutorial on Regularized Partial Correlation Networks. Psychological Methods. 23(4), 617-634

Evans, D. E., \& Rothbart, M. K. (2007). Developing a model for adult temperament. Journal of Research in Personality, 41(4), 868-888. doi:10.1016/j.jrp.2006.11.002 
Faelens, L., Hoorelbeke, K., Fried, E., De Raedt, R., \& Koster, E.H.W. (2019). Negative influences of Facebook use through the lens of network analysis. Computers in Human Behavior, 96, 13-22.

Friedman, J. H., Hastie, T., \& Tibshirani, R. (2014). glasso: Graphical lasso- estimation of Gaussian graphical models. R package version 1.8

Frison E. \& Eggermont S. (2015). Toward an Integrated and Differential Approach to the Relationships Between Loneliness, Different Types of Facebook Use, and Adolescents' Depressed Mood. Communication Research. 1-28.

Frison, E., \& Eggermont, S. (2016). Exploring the relationships between different types of Facebook use, perceived online social support, and adolescents' depressed mood. Social Science Computer Review, 34(2), 153-171.

Fruchterman, T., \& Reingold, E. (1991). Graph drawing by force-directed placement. Software: Practice and Experience, 21, 1129-1164.

Gibbons F.X., Buunk B.P. (1999) Individual differences in social comparison: Development of a scale of social comparison orientation. Journal of Personality and Social Psychology, $76,129-142$

Gupta, N., \& Irwin, J. D. (2016). In-class distractions: The role of Facebook and the primary learning task. Computers in Human Behavior, 55, 1165-1178. doi:10.1016/j.chb.2014.10.022

Haslbeck, J. M. B., \& Fried, E. I. (2017). How predictable are symptoms in psychopathological networks? A reanalysis of 18 published datasets. Psychological Medicine, 47, 27672776.

Haslbeck, J. M. B., \& Waldorp, L. J. (2016). mgm: Estimating time-varying mixed graphical models in high-dimensional data. R package version 1.2-4. 
Indian, M., \& Grieve, R. (2014). When Facebook is easier than face-to-face: Social support derived from Facebook in socially anxious individuals. Personality and Individual Differences, 59, 102-106.

Joormann, J., Talbot, L., \& Gotlib, I. H. (2007). Biased processing of emotional information in girls at risk for depression. Journal of abnormal psychology, 116(1), 135.

Joormann, J., \& Gotlib, I. H. (2010). Emotion regulation in depression: Relation to cognitive inhibition. Cognition and Emotion, 24(2), 281-298.

Joormann, J., \& Stanton, C. H. (2016). Examining emotion regulation in depression: A review and future directions. Behaviour Research and Therapy, 86, 35-49.

Junco, R., \& Cotten, S. R. (2011). Perceived academic effects of instant messaging use. Computers \& Education, 56(2), 370-378.

Junco, R., \& Cotten, S. R. (2012). No A 4 U: The relationship between multitasking and academic performance. Computers \& Education, 59(2), 505-514.

Junco, R. (2015). Student class standing, Facebook use, and academic performance. Journal of Applied Developmental Psychology, 36, 18-29.

Koster, E. H., De Lissnyder, E., Derakshan, N., \& De Raedt, R. (2011). Understanding depressive rumination from a cognitive science perspective: The impaired disengagement hypothesis. Clinical psychology review, 31(1), 138-145.

Kirschner, P. A., \& Karpinski, A. C. (2010). Facebook and academic performance. Computers in Human Behavior, 26(6), 1237-1245.

Lovibond P.F., Lovibond, S.H. (1995). The structure of negative emotional states: Comparison of the Depression Anxiety Stress Scales (DASS) with the Beck Depression and Anxiety Inventories. Behaviour Research and Therapy, 33, 335-343.

Lyubomirsky, S., King, L. A., \& Diener, E. (2005). The benefits of frequent positive affect: Does happiness lead to success? Psychological Bulletin, 131, 803-855. 
Min, J. (2017). Effects of the use of social network sites on task performance: Toward a sustainable performance in a distracting work environment. Sustainability, 9(12), 2270.

Ophir, E., Nass, C., \& Wagner, A. D. (2009). Cognitive control in media multitaskers. Proceedings of the National Academy of Sciences, 106(37), 15583-15587.

Paradise AW, Kernis, MH. (1999). Development of the Contingent Self-esteem Scale. Unpublished data, University of Georgia.

Phua, J., \& Jin, S. A. A. (2011). 'Finding a home away from home': the use of social networking sites by Asia-Pacific students in the United States for bridging and bonding social capital. Asian Journal of Communication, 21(5), 504-519.

Putnam, R. (2000). Bowling alone: Collapse and revival of American community. New York: Simon \& Schuster.

Reis, H. T., Sheldon, K. M., Gable, S. L., Roscoe, J., \& Ryan, R. M. (2018). Daily well-being: The role of autonomy, competence, and relatedness. In Relationships, Well-Being and Behaviour (pp. 317-349). Routledge.

Rosenberg, M. (1965). Society and the adolescent self-image. Princeton, NJ: Princeton University Press.

Rosen, L. D., Mark Carrier, L., \& Cheever, N. A. (2013). Facebook and texting made me do it: Media-induced task-switching while studying. Computers in Human Behavior, 29(3), $948-958$.

Sanbonmatsu, D. M., Strayer, D. L., Medeiros-Ward, N., \& Watson, J. M. (2013). Who MultiTasks and Why? Multi-Tasking Ability, Perceived Multi-Tasking Ability, Impulsivity, and Sensation Seeking. PLoS ONE, 8(1), e54402.

Sherman, A. M., Lansford, J. E., \& Volling, B. L. (2006). Sibling relationships and best friendships in young adulthood: Warmth, conflict, and well-being. Personal Relationships, 13(2), 151-165. 
Sousa-Poza, A., \& Sousa-Poza, A. A. (2000). Well-being at work: a cross-national analysis of the levels and determinants of job satisfaction. The journal of socio-economics, 29(6), $517-538$.

Sowislo, J. F., \& Orth, U. (2013). Does low self-esteem predict depression and anxiety? A metaanalysis of longitudinal studies. Psychological Bulletin, 139(1), 213-240. doi:10.1037/a0028931

Steers, M. N. (2016) 'It's complicated': Facebook's relationship with the need to belong and depression. Current Opinion in Psychology, 9, 22-26.

Steers M.N., Wickham R.E., Acitelli L.K. (2014). Seeing everyone else's highlight reels: How Facebook usage is linked to depressive symptoms. Journal of Social and Clinical Psychology, 33, 701-731.

Su, C. C., \& Chan, N. K. (2017). Predicting social capital on Facebook: The implications of use intensity, perceived content desirability, and Facebook-enabled communication practices. Computers in Human Behavior, 72, 259-268.

Treynor W,. Gonzalez R., \& Nolen-Hoeksema, S. (2003). Rumination reconsidered: A psychometric analysis. Cognitive therapy and research, 27, 247-259.

Verduyn, P., Ybarra, O., Résibois, M., Jonides, J., \& Kross, E. (2017). Do social network sites enhance or undermine subjective well-being? A critical review. Social Issues and Policy Review, 11(1), 274-302.

Vogel E.A., Rose, J.P. (2016) Self-reflection and interpersonal connection: Making the most of self-presentation on social media. Translational Issues in Psychological Science; 2, 94-302.

Vogel, E. A., Rose, J. P., Roberts, L. R., \& Eckles, K. (2014). Social comparison, social media, and self-esteem. Psychology of Popular Media Culture, 3(4), 206-222. 
Watson, D., \& Clark, L. A. (1994). Emotions, moods, traits, and temperaments: Conceptual distinctions and empirical findings. In P. Ekman \& R. J. Davidson (Eds.), The nature of emotion: Fundamental questions. New York: Oxford University Press.

Woods, H. C., \& Scott, H. (2016). \#Sleepyteens: Social media use in adolescence is associated with poor sleep quality, anxiety, depression and low self-esteem. Journal of Adolescence, 51, 41-49.

Wouters, S., Duriez, B., Luyckx, K., Klimstra, T., Colpin, H., Soenens, B., \& Verschueren, K. (2013). Depressive symptoms in university freshmen: Longitudinal relations with contingent self-esteem and level of self-esteem. Journal of Research in Personality, 47(4), 356-363

Zhao, T., Li, X., Liu, H., Roeder, K., Lafferty, J., \& Wasserman, L. (2015). huge: HighDimensional undirected graph estimation. R package version 1.2.7. 